\title{
Relationship Between Pathological Response and Molecular Subtypes in Locally Advanced Breast Cancer Patients Receiving Neoadjuvant Chemotherapy
}

\section{EZGI DEĞERLI ( $\square$ ezgitastan.19@gmail.com)}

Cerrahpasa Medical School: Istanbul Universitesi-Cerrahpasa Cerrahpasa Tip Fakultesi https://orcid.org/0000-0002-8664-5701

Nihan Şentürk Öztaş

İstanbul Üniversitesi-Cerrahpaşa: Istanbul Universitesi-Cerrahpasa Gülin Alkan

İstanbul Üniversitesi-Cerrahpaşa: Istanbul Universitesi-Cerrahpasa

\section{Şahin Bedir}

İstanbul Üniversitesi-Cerrahpaşa: Istanbul Universitesi-Cerrahpasa

\section{Sümeyra Derin}

İstanbul Üniversitesi-Cerrahpaşa: Istanbul Universitesi-Cerrahpasa

\section{Nahıda Valıkhanova}

İstanbul Üniversitesi-Cerrahpaşa: Istanbul Universitesi-Cerrahpasa

\section{Betül Saraç}

İstanbul Üniversitesi-Cerrahpaşa: Istanbul Universitesi-Cerrahpasa

Nebi Serkan Demirci

İstanbul Üniversitesi-Cerrahpaşa: Istanbul Universitesi-Cerrahpasa

Hulusi Fuat Demirelli

İstanbul Üniversitesi-Cerrahpaşa: Istanbul Universitesi-Cerrahpasa

Hande Turna

İstanbul Üniversitesi-Cerrahpaşa: Istanbul Universitesi-Cerrahpasa

\section{Research Article}

Keywords: breast cancer molecular subtypes, neoadjuvant chemotherapy, complete pathological response

Posted Date: October 29th, 2021

DOI: https://doi.org/10.21203/rs.3.rs-1021727/v1 
License: (c) (i) This work is licensed under a Creative Commons Attribution 4.0 International License. Read Full License

Version of Record: A version of this preprint was published at Journal of Chemotherapy on February 26th, 2022. See the published version at https://doi.org/10.1080/1120009X.2022.2043514. 


\section{Abstract}

Background: More than half of patients with breast cancer were diagnosed with locally advanced stages of the disease (54\%). This study aimed to explain the pathological response received with neoadjuvant chemotherapy (NACT) according to the molecular classification of breast cancer in patients with locally advanced tumors.

Methods: One hundred one patients with locally advanced breast cancer treated with neoadjuvant chemotherapy were analyzed. Patients were classified into five molecular subtypes based on the profile of the estrogen receptor, progesterone receptor, HER2, and Ki-67. We determined associations between pathologic complete response and molecular subgroups.

Results: Most patients had luminal A tumors ( $\mathrm{n}: 28,27.7 \%)$. The overall rate of pathological complete response (pCR) was $34.7 \%$ (n:35). Tumors that presented the highest rate of pCR were pure HER2positive, at $60 \%(\mathrm{n}: 6 ; \mathrm{OR}, 3.2 ; 95 \% \mathrm{Cl}, 0.8-12.2)$. According to logistic regression analysis, the factors affecting PCR were HER2 positivity and clinically positive axilla before NACT. Luminal A tumors had a significantly lower $\mathrm{pCR}$ rate. (7.1\%,p: 0.001). Despite the low $\mathrm{pCR}$ rate, luminal $A$ tumor survival was the best subgroup $(p<0.001)$. However, there was no difference between EFS and OS according to $p C R$ in any molecular subgroups.

Conclusion: Pathological complete response is directly related to the subtypes of breast cancer. A high rate of complete pathological response is observed in the pure HER2-positive group. However, EFS and OS were not statistically significant in patients with and without $\mathrm{PCR}$.

\section{Introduction}

Neoadjuvant chemotherapy is an option for patients with early and advanced breast cancer when an indication for chemotherapy is given. The main goal in patients given neoadjuvant chemotherapy(NACT) is to provide a complete pathological response(pCR) [1]. The pathological response of breast cancer to neoadjuvant chemotherapy is a prognostic indicator for long-term disease-free and overall survival [2]. Pathological complete response means that invasive tumor does not remain microscopically in the breast tissue after neoadjuvant chemotherapy. Pathological complete response is observed in $10-40 \%$ of breast cancer patients [3]. Many factors affect this different ratio. Incidence and prognostic impact of pCR vary among breast cancer subtypes. Molecular subtypes of breast cancer give different responses to neoadjuvant chemotherapy [4]. Breast cancer is divided into five significant molecular subtypes based on the absence or presence of molecular markers for estrogen or progesterone receptors and human epidermal growth factor 2(ERGF2 or HER2). Hormone receptor-positive/HER2 negative (70\% of patients have included two subtypes (Luminal A had low Ki-67 score, luminal B had high Ki-67 score), pure HER2positive (15\%-20\%), and triple-negative (15\%) [5]. In our study, to differentiate luminal A and luminal B HER2-negative tumors, the cutoff point for the Ki-67 marker was 15\%. Luminal A subtype responds poorly 
to chemotherapy, but its prognosis is the best among others. A triple-negative tumor has the worst prognosis. Fortunately, triple-negative breast cancer has a higher response to chemotherapy [6].

There are several histopathological classifications to categorize the tumor response to NACT [7]. We use the Miller-Payne grading (MPG) system to determine the pathological response of NACT. In our pathologist's opinion Miller-Payne histological grading system was most useful. MPG provides a five-step scale based on tumor cellularity in the lumpectomy or mastectomy specimen compared with the pretreatment core biopsy. MPG is divided into five grades. Grade 1, pathological no response(pNR); grade 2 , minor loss of cellularity (30\%) grade 3 , estimated $30 \%-90 \%$ reduction in tumor cells; grade $4,90 \%$ loss of tumor cells; and grade 5, correct pathological response(pCR) means no invasive carcinoma but ductal carcinoma in situ may be present [8].

This study aimed to determine the pathological response and prognosis following neoadjuvant chemotherapy in the molecular subtypes of breast cancer in patients with locally advanced tumors treated within Istanbul University-Cerrahpaşa, Cerrahpaşa Medical Faculty Oncology Department.

\section{Methods}

Ethics approval was obtained from Istanbul University Cerrahpaşa-Cerrahpaşa Medical Faculty, Turkey, at the beginning of the study. We evaluated 101 patients diagnosed between 2016 and 2020 by true-cut biopsy and treated with NACT followed by surgical management. Patients who progressed to clinical stage IV, those who for some reason did not receive surgical management after neoadjuvant therapy, and patients whose pathology report cannot be reached were excluded (figure 1). We obtained information about the clinic and demographic data of all enrolled patients from the hospital database. The variables analyzed in our study were age, body mass index, menopausal status, histopathological characteristics of the biopsy, initial clinical stage of the disease, NACT regimen received, clinical response, type of surgical management, pathological response according to Miller-Payne grading, adjuvant treatment if offered, presence of recurrence and disease progression, overall survival.

The NACT regimens comprised 5-fluorouracil, epirubicin, and cyclophosphamide and docetaxel (FEC-T); F $600 \mathrm{mg} / \mathrm{m} 2$ intravenously on day $1, E 60 \mathrm{mg} / \mathrm{m} 2$ intravenously on day 1 , and C $600 \mathrm{mg} / \mathrm{m} 2$ intravenously on day 1, repeated every 21 days for 4 or 6 cycles. Patients who received four cycles of FEC received an additional four cycles of docetaxel $75 \mathrm{mg} / \mathrm{kg}$ ); adriamycin, cyclophosphamide, and docetaxel or weekly paclitaxel (ACT, A $60 \mathrm{mg} / \mathrm{m} 2$ intravenously on day 1, C $600 \mathrm{mg} / \mathrm{m} 2$ intravenously on day 1 for four cycles after that $T 75 \mathrm{mg} / \mathrm{m} 2$ intravenously on day 1 for four cycles of weekly paclitaxel for 12 weeks. Patients with HER2-positive $\mathrm{H} 6 \mathrm{mg} / \mathrm{kg}$, following 8mg/kg loading dose intravenously on day 1, repeated every 21 days). Trastuzumab(H) was given as adjuvant therapy for four cycles in HER-2 positive cases (ACTH or FEC-TH). 13 cycles were given after the operation. In recent years, pertuzumab has been used in neoadjuvant therapy in HER2-positive patients, and six patients were able to receive pertuzumab in addition to trastuzumab (ACTHP). Carboplatin AUC2 weekly treatment was added to the ACT regimen in some patients with the triple-negative subtype. Twenty-three patients underwent eight ACT cycles; five 
patients received ACT-H, two patients received ACT plus carboplatin, six patients received six cycles FEC, forty patients received FEC-T, and fourteen patients received FEC-TH chemotherapy regimens. Three patients who were not eligible to take anthracycline received docetaxel and trastuzumab, and two patients received only weekly paclitaxel.

Lumpectomy or mastectomy was performed in patients whose neoadjuvant chemotherapy was completed. The axillary approach was divided into two groups as sentinel lymph node biopsy or axillary curettage. The patients were divided into five groups according to their molecular subtypes after operation: Luminal A, Luminal B HER2-negative, Luminal B HER2-positive, Pure HER2-positive, Triple negative. Luminal A tumor ER, PR positive, HER2 negative, Ki-67 less than 15\%, Luminal B ER, PR positive, Ki-67 above 15\% were defined. Luminal B itself was examined in 2 groups: HER2 positive and HER2 negative. Pure-HER2 is ER, PR negative, HER2 positive; triple-negative ER, PR, HER2 negative subgroups. He defined the cut-off value as $1 \%$ to mean ER and PR positive. A positive Ki-67 finding was defined as $>15 \%$, and a negative finding was defined as $\leq 15 \%$. HER2 scores of 0 and $1+$ were considered harmful, $3+$ was means HER2-positive. When a score of $2+$ was found, additional fluorescent in situ hybridization testing was performed to determine the HER2 gene amplification status.

\section{Statistical analysis}

SPSS 15.0 for Windows program was used for statistical analysis. Descriptive statistics; numbers and percentages were given for categorical variables, and numerical variables were given as mean, standard deviation, minimum, maximum, median, interquartile range. The rates in the groups were compared with the Chi-Square Test. When the numeric variables did not meet the normal distribution condition, more than two independent group comparisons were made using the Kruskal Wallis Test. Two independent group comparisons were made with the Mann-Whitney U Test. In more than two groups, subgroup analyses were performed with Mann Whitney U Test and interpreted with Bonferroni correction. Two independent group comparisons of the numerical variable satisfying the normal distribution condition were compared with Student's t-test. Logistic Regression Analysis analyzed determining factors. Statistical alpha significance level was accepted as $p<0.05$. The EFS and OS functions were determined with the Kaplan-Meier estimator.

\section{Results}

One hundred thirty-five patients who received neoadjuvant chemotherapy between January 2016 and January 2020 were included in our study. Of the 135 patients with received neoadjuvant treatment, 34 patients were excluded for the following reasons: 20 (14\%) patients had missing data, 9(8\%) patients were restaged because of distant metastasis, and 5 (4\%) patients were withdrawn for surgery.

In total, 101 patients were included (median age, $46,7 \pm 10,9$ years; range, $24-73$ ); $55.4 \%$ (n:56) of the patients were premenopausal, $33.7 \%$ (n:34) of the patients were postmenopausal. The tumor was located in the left breast in $59(58.4 \%)$ patients, in $41(40.6 \%)$ in the right breast, and in the bilateral breast in 
1(1\%) patient. There was no significant correlation between age, menopausal status, tumor location, and complete pathological response.

We examined the patients in five groups according to their body mass index. Mean body mass index 27.9 \pm 5.4 (range 17-43). There was no difference between those with and without a complete pathological response (p: 0.23).

Most patients were classified in clinical stage IIIB $(30.7 \%, n=31)$ in clinical stage. The most frequent tumor histological type was the invasive ductal carcinoma identified in 85 patients (84.2\%). 51 (50.5\%) patients had size T2 tumors, and 48(47.5\%) patients had clinical N2. The most common histological grade 2 tumor was found in true-cut biopsies $(60.4 \%, n=61)$. (table1)

Patients were divided into five groups according to molecular subtypes. Luminal A tumors were $27.7 \%$ of cases, with 28 patients; luminal B HER2-negative was described in 25 patients (24.8\%); 10 patients presented pure HER2-positive tumors (9.9\%); and the triple-negative tumors reached $18.8 \%$, with 19 patients, luminal B HER2 positive was $18.8 \%$, with 19 patients.

The overall $p C R$ rate was $34.7 \%(n=35)$. The distribution of patients' pathological response assessment according to the MPG system was as follows: n:2(1.9\%) in MPG 1, n:35 (34.7\%) in MPG 2, n:16 (15.8\%) in MPG 3, n: 13 (15.8\%) in MPG 4 and n:34(\%34.7) in MPG 5. MPG 5 represents the pCR. Taking luminal A tumors as a reference category, it was found that tumors with the highest pCR rate were pure HER2positive tumors, at $60 \%(n=6 ; O R, 3.2 ; 95 \% \mathrm{Cl}, 0.8-12.2)$. pCR was $57.8 \%(\mathrm{n}=11 ; \mathrm{OR}, 3,3 ; 95 \% \mathrm{Cl}, 1.1-9.2)$ in Luminal B HER2-positive tumor, $44 \%(n=11 ; O R, 1.7 ; 95 \% \mathrm{Cl}, 0.6-4.2)$ in Luminal B HER2-negative tumor, and $21 \%(n=5)$ in triple-negative tumor (table 2$)$. Luminal A $(7.1 \%)$ had the lowest pathological complete response rate ( $\mathrm{p}: 0.001)$. According to univariate logistic regression analysis, complete response was $93 \%$ less in Luminal A tumors (OR, 0.072; $95 \% \mathrm{Cl}, 0.015-0.34)$.

The axilla was clinically positive in $88.6 \%$ of the 35 patients with the complete pathological response. A fine-needle aspiration biopsy confirmed each patient with clinically positive axilla. According to the univariate regression analysis, invasive tumor in the axilla positively affects the chemotherapy response (p: 0.043 , OR: $3.69 ; 95 \% \mathrm{Cl} 1.0-13.1$ ). It was observed that $57 \%$ of the patients with clinically positive axilla were in the group with a high response. (Luminal B HER2 positive, luminal B HER2 negative, and pure HER2-positive groups).

The highest rate of pCR was evidenced in grade 3 tumors, at $46.2 \%(n=18)$. However, no significant relationship was found between molecular subtypes and pathological complete response and grade ( $\mathrm{p}$ : $0.67)$.

The index Ki-67 in the entire patient population was found to be $39.4 \pm 24.9$ (range 5-90). The median index of Ki-67 was 40 (range 30-60) in patients with the complete pathological response and 30 (15-60) patients with no complete pathological response. There was no statistically significant difference between the two groups (p:0.06). 
Anthracycline-based chemotherapy was the most administered neoadjuvant therapy ( $n=96,95 \%$ ). Taxane-based chemotherapy was given to $5(5 \%)$ patients who could not receive anthracycline. Trastuzumab was added to the treatment in positive patients for HER2( $n=22,28.8 \%)$. Pertuzumab + trastuzumab was used in 6 patients. Carboplatin is added to $2(2 \%)$ treatments with triple-negative tumors. The adriamycin + cyclophosphamide - taxane (ACT) regimen achieved the highest pCR rate, 29.9\% (n:26), and it was similar to FEC and FETCH. Adjuvant chemotherapy was administered to 9 (8.9\%) patients and most of them with triple-negative tumors (n:7,77\%) with evidence of residual tumor in the surgical specimen. Capecitabine was the most preferred in adjuvant therapy. (n:5, 56\%). In the adjuvant treatment, docetaxel was preferred for the patients who did not receive taxane-based therapy in neoadjuvant therapy (n: 4, 44\%). All of them with positive hormone receptors received adjuvant hormone therapy $(\mathrm{n}: 73,74.3 \%)$ and $91 \%(\mathrm{n}: 90)$ of patients received adjuvant radiotherapy. The most preferred hormonotherapy was letrozole, as $34 \%$ of the patients were postmenopausal (table 3 ).

Clinical response was given to neoadjuvant therapy. The majority of patients with clinical responses were constituted (n:89, 88,1\%). 35 (39.2\% clinically responding patients also had a complete pathological response.

Lumpectomy management was performed in $22.8 \%$ (n:23) of patients, and mastectomy was performed in $78(77.2 \%)$ patients. Because their tumors were locally advanced, 80 patients $(79.2 \%)$ were taken to axillary lymphadenectomy. Sentinel lymph node biopsy was performed in $21(20.8 \%)$ patients. Invasive ductal carcinoma accounted for the majority of all tumors. (n:85, 84\%). The pathological complete response rate in ductal carcinoma was $37 \%$ (n:31) (table 3). The complete pathological response could not be achieved in any tumors with clear cell, metaplastic, neuroendocrine, and squamous differentiation.

Tumor markers were examined before and after neoadjuvant in the patient. The initial CEA level was 3.00 \pm 1.94 (in the range of 0.5-9), and the Ca15.3 level was $28.3 \pm 23.1$ (6-184). After neoadjuvant therapy, the CEA level was $1.81 \pm 1.16$ (range 0.3-5), and the Ca15.3 level was $14.9 \pm 8.7$ (range 3.4-60). The mean initial CEA of the pure HER2-positive group was significantly higher in the subgroup analysis than Luminal B HER2-positive groups (p: 0.003). However, there was no significant difference between initial tumor marker and post-neoadjuvant tumor marker in groups with and without complete pathological response.

The overall recurrence rate (local and distant) was 14\% $(n=14)$, and the median recurrence time of 24 months (6-44 months) with a follow-up of 60 months. Local or regional recurrence was n:3 (21\%), and the number of distant metastases was $n=11(79 \%)$. Two of the patients $(67 \%)$ with local recurrence were triple-negative. The other patient was Luminal B HER2-positive. None of the patients with local recurrence had a complete pathological response. The pathological response of all these patients was MPG 2. The molecular subtype that determined the most relapses was luminal B HER2-positive, at $36 \%(n=5)$, followed by the triple-negative at $29 \%(n=4)$, luminal B HER2-negative,21\% (n:3), and pure HER2-positive presented two cases (14\%). Of the 11 who developed distant metastases, $36 \%(n=4)$ had a complete 
pathological response. Most recurrences $(71.5 \% ; n=10)$ occurred in patients in that no pathological response was obtained (table 4). The most common site of metastasis was the first bone (7\%), the second lung (6\%).

A total of 5 deaths were documented, describing $5 \%$ of the total patients, with a median follow-up time of 19 months (6-31 months). Among these, the number of patients who died despite a complete pathological response was n: $2(40 \%)$. Two of these patients were HER2-positive. Luminal A subtype had significantly higher overall survival $(p<0.001)$. EFS and OS according to $p C R$ in molecular subgroups were evaluated with non-parametric Kaplan-Meier estimator. However, there was no difference between EFS and OS according to PCR in any molecular subgroups.

Table 1. Demographic and clinical characteristics of patients 
Characteristics

Median age (range), yr
Total of patients $(n=101), n(\%)$

$46,7 \pm 10,9(24-73)$
Menopousal status

Premenopousal

Perimenopousal

Postmenopousal
$56(55,4)$

$11(10,9)$

$34(33,7)$

Body mass index, $\mathrm{kg} / \mathrm{m} 2$

Low weight (<18)

$1(1,0)$

Normal (18-25)

$35(34,7)$

Overweight (25.1-29.9)

$28(27,7)$

Class I obesity (30-34.9)

$28(27,7)$

Class II obesity (35-39.9)

$5(5,0)$

Class III obesity $(>40)$

$4(4,0)$

Tumor histology

Ductal carcinoma

$85(84,2)$

Lobuler carcinoma

$3(3,0)$

Others

$13(12,8)$

Tumor localization

Left

$59(58,4)$

Right

$41(40,6)$

Bilaterale

$1(1,0)$

Tumor size

T1

T2

T3
$13(12,9)$

$51(50,5)$

$15(14,9)$ 
Lymph node involvement

N1

N2

N3

Clinical stage

1

2A

2B

$3 \mathrm{~A}$

3B

3C

Tumor grade

1

2

3

Estrogen receptors

Positive

Negative

Progesterone receptors

Positive

Negative

Human epidermal growth factor 2

Positive

Negative

Ki-67

$\leq 15$

$>15$
$74(73,3)$

$30(29,7)$

$48(47,5)$

$23(22,8)$

$4(4,0)$

$16(15,8)$

$7(6,9)$

23(22,8)

$31(30,7)$

$20(19,8)$

$1(1,0)$

$61(60,4)$

$39(38,6)$

$27(26,7)$

$68(67,3)$

$33(32,7)$

$29(28,7)$

$72(71,3)$

$30(29,7)$

$71(70,3)$ 
Table 2. Miller-Payne grade 5 (pCR) with molecular subtypes

\begin{tabular}{|c|c|c|c|c|c|c|c|}
\hline \multirow[t]{2}{*}{ Moleculer subtypes } & \multicolumn{2}{|c|}{ Total } & \multicolumn{2}{|c|}{ PCR } & \multicolumn{2}{|c|}{ Non-pCR } & \multirow{2}{*}{$\begin{array}{l}\text { P-value } \\
0.001\end{array}$} \\
\hline & $\mathrm{n}$ & $\%$ & $\mathrm{n}$ & $\%$ & $\mathrm{n}$ & $\%$ & \\
\hline Luminal A & 28 & 27,7 & 2 & 5,7 & 26 & 39,4 & \\
\hline Luminal B/ HER2+ & 19 & 18,8 & 11 & 14,3 & 8 & 12,1 & \\
\hline Luminal B/HER2- & 25 & 24,8 & 11 & 31,4 & 14 & 21,2 & \\
\hline Pure HER2+ & 10 & 9,9 & 6 & 17,1 & 4 & 6,1 & \\
\hline Triple negative & 19 & 18,8 & 5 & 14,3 & 14 & 21,2 & \\
\hline
\end{tabular}

Table 3. Treatments administered to patients 


\begin{tabular}{|ll|}
\hline Treatment & Total of patients, $\mathrm{n}(\%)$ \\
\hline Neoadjuvant chemotherapy regiment (n:101) & \\
ACT & $40(39,6)$ \\
FECTH & $23(22.8)$ \\
FEC & $14(13,9)$ \\
ACTHP & $6(5,9)$ \\
ACTH & $6(5,9)$ \\
ACTK & $5(5)$ \\
Others & $2(2)$ \\
& $5(5)$ \\
\hline Surgical treatment & \\
Mastectomy & \\
Conserving surgery & $78(77,2)$ \\
\hline Adjuvant treatments received & $23(22,8)$ \\
\hline Hormonotherapy & \\
\hline Axillary approach & \\
\hline RLNB & \\
\hline
\end{tabular}

Table 4: Frequency of recurrence and metastasis by molecular subtypes 


\begin{tabular}{llllll} 
Recurrence & $\begin{array}{l}\text { Luminal } \\
\text { A }\end{array}$ & $\begin{array}{l}\text { Luminal } \\
\text { B/HER2- }\end{array}$ & $\begin{array}{l}\text { Luminal B/ } \\
\text { HER2+ }\end{array}$ & $\begin{array}{l}\text { Pure } \\
\text { HER2+ }\end{array}$ & $\begin{array}{l}\text { Triple } \\
\text { negative }\end{array}$ \\
\hline $\begin{array}{l}\text { Total } \\
\text { (n:14,\%14) }\end{array}$ & 0 & $3(21)$ & $5(36)$ & $2(14)$ & $4(29)$ \\
\hline $\begin{array}{l}\text { Local } \\
\text { (n:3, 21\%) }\end{array}$ & 0 & 0 & $1(33)$ & 0 & $2(67)$ \\
\hline $\begin{array}{l}\text { Distant } \\
\text { (n:11,79\%) }\end{array}$ & 0 & $3(27)$ & $4(36)$ & $2(18)$ & $2(18)$ \\
\end{tabular}

\section{Discussion}

In 2020, there were 2.3 million women diagnosed with breast cancer and 685000 deaths globally. According to statistical data, approximately $54 \%$ of these patients are locally advanced [9]. Neoadjuvant chemotherapy is the standard treatment for locally advanced breast cancer. It is also recommended for breast-conserving surgery and minimal axillary lymph node dissection in early-stage breast cancer[10]. However, the first aim of neoadjuvant treatment is to downstage the disease and render in-operable tumors resectable. The pathological target in neoadjuvant therapy is the complete pathological response(pCR) [11]. pCR is a prognostic factor and linked to a better overall survival (OS) and event-free survival (EFS)[12]. The most important factor affecting the complete pathological response is the affinity of the tumor to neoadjuvant therapy. The neoadjuvant chemotherapy sensitivity of the tumor is different in molecular subtypes[13]. Molecular subtypes are divided into five main groups depending on the presence or absence of estrogen, progesterone, HER2 receptor, and the percentage of Ki-67[14]: Luminal A, Luminal B HER2-negative, Luminal B HER2-positive, Pure HER2-positive, Triple-negative or basal-like. Luminal A tumors are represented in 30-40\%, luminal B HER2-negative tumors 20-30\%, HER2-positive tumors (pure HER2-positive and Luminal B) 15-20\%, and triple-negative tumors 15-20\%. In our data, the incidence of subtypes was similar. However, luminal A tumor was less than the literature, with a rate of $27.7 \%$.

Pathologic responses of primary breast tumor were evaluated according to the Miller-Payne grading system, which defines pCR as the absence of invasive and in situ carcinoma in the breast and axillary nodes (ypT0/ypN0) [15]. The clinical trials have shown that the complete pathological response is related to good treatment results[16]. In recent years, factors predicting complete pathological response were investigated. ER, PR levels, HER2 status, tumor grade, molecular subtype, perineural invasion, vascular invasion, Ki-67, and tumor size could not be fully correlated with pathological response[17]. Grade 5 in the MPG system was considered to complete pathological response. In our study, the number of patients with complete pathological responses was 35. (34.7\%). In the 101 patients, pathological complete response rates for primary tumour in luminal A, luminal B/HER2-, luminal B/HER2+, pure HER2-positive, and triple- 
negative disease were $7.1 \%, 44.0 \%, 57.8 \%, 60.0 \%$, and $21.0 \%$, respectively. In other words, the group with the highest pathological response rate was pure HER2 positive, and the group with the lowest was luminal $A(\% 7,1)$. $P C R$ rate was significantly lower for luminal $A$ tumor $(P=0.001)$. Even if the pathological complete response rate of luminal A tumors is low, survival rates are high. No local recurrence or death was observed in any of the Luminal A tumors in our data. Although luminal A tumors respond less to chemotherapy, the positivity of ER, PR, and low Ki-67 level is associated with a good prognosis [18]

The pathological complete response rates in lymph nodes of luminal A, luminal B/HER2-, luminal B/HER2+, pure HER2-positive, and triple-negative disease were $14.3 \%, 52.0 \%, 89.5 \%, 60.0 \%$, and $47.4 \%$, respectively. There was a statistically significant difference in axillary response rates in the molecular subtype groups $(p<0.001)$.

HER2-positive tumors are known for their aggressive behavior. In the study of Buzdar et al., the pCR rate for HER2-positive tumors was given as 66.7\%[19]. Our study examined the HER2-positive tumors in 2 categories: Luminal B HER2-positive and pure HER2-positive. Pathological complete responses of these two subgroups were compatible with the literature, $57.8 \%$, and $60 \%$, respectively. When the patients with a pathologically complete response and those who did not were compared, a significantly higher HER2positivity was found at complete responders $(p=0.017)$. Anti-HER2 treatments contributed to this critical response in neoadjuvant therapies. In a meta-analysis by Antonis et al., they determined that the addition of trastuzumab in HER2-positive breast cancer in the neoadjuvant setting improves the probability of achieving higher pCR[20]. In addition, clinical studies have shown that the use of dual HER2 blockade (pertuzumab + trastuzumab) in neoadjuvant therapy further increases the pCR rate[21]. Clinical studies have shown that neoadjuvant chemotherapy with trastuzumab plus pertuzumab increases the $\mathrm{PCR}$ rate compared with trastuzumab alone, resulting in about 50-70\% pCR rate[22]. In our data, pCR was observed in all six patients who received dual HER2 blockade. The low number of patients given pertuzumab is that it was not paid by health insurance before 2019 .

Triple-negative tumors are also tumors with an aggressive course. However, it has a high sensitivity to chemotherapy. Neoadjuvant chemotherapy is seen as the standard treatment for triple-negative and HER2-positive tumors. In the study of Sharma et al., 35-55\% pCR was seen with neoadjuvant cytotoxic chemotherapy in triple-negative tumors[23,24]. In the GeparSixto study, 53.2\% pCR was obtained by adding carboplatin to neoadjuvant therapy in triple-negative tumors[25]. In our study, the rate of pCR in triple-negative tumors was much lower than in the literature $(21 \%, n: 5)$. The neoadjuvant treatment of patients with a complete response was combined-based therapy with anthracycline and a taxane (FEC, $\mathrm{ACT}$, or FECT). Carboplatin was added to the treatment of only one of these patients. When all triplenegative patients were examined, there were already only two patients to whom carboplatin was added to their treatment. In other words, $50 \%$ of the patients who added carboplatin in our study gave a complete response.

Luminal A tumor is the least chemosensitive; our study achieved pCR rates of 7,1\% (n:2). In the study of Collins et al., 114 patients with Luminal A tumor had a pCR rate of 7.9\%[26]. In Collins et al. study, 
patients with complete pathological responses were patients with high-grade tumors and weak PR expression. In our study, the tumor grade of 2 patients with complete pathological response was two, and the PR expression percentage was below $10 \%$. Similar results were obtained for luminal A tumors in the 14000 diseases neoadjuvant study of Haque et al. The rate of pCR in patients with luminal A tumor was found to be $0.03 \%$. However, in the 5-year overall survival analysis, Luminal A tumors are much better than other subtypes. (98.2\%) [27]. This shows that the survival of luminal A tumors is quite good, although they do not give a complete pathological response. In our study, no recurrence was observed in any patient with luminal $A$ tumor despite the low $\mathrm{PCR}$ rate.

In our data, the second-highest pCR rate was in Luminal B HER2-positive tumor $(\% 57,8)$. In the study of Wang et al., The rate of pCR was $13.4 \%$ in patients with Luminal B tumors. However, Wang et al. showed that patients with a high $\mathrm{Ki}-67$ index above $40 \%$ had a better pathological complete response and recurrence-free survival[28]. A meta-analysis of 6793 patients by Tao et al. showed Ki-67 labeling index is a predictive marker for a complete pathological response to neoadjuvant chemotherapy in breast cancer[29]. In our data, the median index of Ki-67 of all patients with a complete pathological response was $40 \%$ (30-65), and the median index of Ki-67 for those who did not have a complete pathological response was 30 (15-60). There was no statistically significant difference between the two groups (p:0.06). However, when patients with luminal B tumors were examined separately, the median index of $\mathrm{Ki}-67$ was 41.7 (range 10-80). The high Ki-67 value of our patients with luminal B tumors may have contributed to a better response to neoadjuvant chemotherapy.

In our data, patients with complete pathological response had a high rate of clinical axilla positivity before neoadjuvant chemotherapy (88.6\%, p: 0.043, OR: 3.69; 95\% Cl 1.0-13.1). Samiei et al. showed that, on the contrary, patients with clinically negative axilla achieved a higher rate of complete pathological response in ER+HER2+, ER-HER2+, and triple-negative subtypes[30]. All patients with clinically positive axilla were in the luminal B HER2-positive and pure HER2-positive groups in our study.

Patients with grade 3 tumor differentiation constituted $38.6 \%$ of the entire population (n:39). The grade 3 group had the highest rate of pCR (n:18, 46.2\%). Diaz-Casas et al. reported the pCR rate as $21.3 \%$ in grade 3 tumors[31]. In the study of Jarzab et al., the pCR rate was reported as $30.9 \%$ in patients with grade 3 nuclear differentiation[32].

Pathological complete response is associated with the neoadjuvant chemotherapy regimen. Anthracycline-based chemotherapy regimens form the basis of treatment. ACT regimen achieved the highest pCR rate, $29.9 \%(n=26)$, and it was similar to FEC and FETCH. In recent years, pertuzumab has been added to the treatment of HER2-positive tumors along with trastuzumab. In addition, carboplatin was added to the anthracycline and taxane regimen in triple-negative tumors. However, since the number of these patients was small in our study, the effects of pertuzumab and carboplatin on pCR could not be reported.

There was a significantly higher EFS and OS difference in the Luminal A subtype compared to the other molecular subtypes. $(p<0.001)$ However, the complete pathological response did not affect survival in 
Luminal A tumor. This can be explained by the low chemosensitivity of Luminal A tumors. Fortunately, long survivals have been achieved with the increase in hormone therapy options over the years. The HER2 positive group had a high pathological complete response. However, this situation was not reflected in EFS and OS in our patient population. Regardless of the subgroup, when the patients were divided into two groups as pathological complete response and non-responder and OS analysis was performed, there was no significant difference in survival between the two groups(p:0.06). However, the short follow-up period in our study may have prevented us from seeing a significant difference. We hope that with a larger patient group and a more extended follow-up period and with the use of new neoadjuvant treatment regimens we can distinguish between pathologically responding and non-pathological subgroups.

\section{References}

1. Spring LM, Fell G, Arfe A, et al. Pathologic Complete Response after Neoadjuvant Chemotherapy and Impact on Breast Cancer Recurrence and Survival: A Comprehensive Meta-analysis. Clin Cancer Res. 2020;26(12):2838-2848. doi:10.1158/1078-0432.CCR-19-3492

2. Simons JM, Jacobs JG, Roijers JP, et al. Disease-free and overall survival after neoadjuvant chemotherapy in breast cancer: breast-conserving surgery compared to mastectomy in a large singlecentre cohort study. Breast Cancer Res Treat. 2021;185(2):441-451. doi:10.1007/s10549-020-05966-y

3. Von Minckwitz G, Untch M, Blohmer J-U et al. Definition and Impact of Pathologic Complete Response on Prognosis After Neoadjuvant Chemotherapy in Various Intrinsic Breast Cancer Subtypes. J Clin Oncol 2012;30:1796-804.

4. Zhao Y, Dong X, Li R et al. Evaluation of the pathological response and prognosis following neoadjuvant chemotherapy in molecular subtypes of breast cancer. Onco Targets Ther 2015;8:151121.

5. Waks AG, Winer EP. Breast Cancer Treatment: A Review. JAMA. 2019;321(3):288-300. doi:10.1001/jama.2018.19323

6. Carey LA, Dees EC, Sawyer L, et al. The triple negative paradox: pri- mary tumor chemosensitivity of breast cancer subtypes. Clin Cancer Res. 2007;13:2329-2334.

7. Blanco Sánchez A, Yébenes L, Berjón A, Hardisson D. Evaluación de la respuesta patológica a quimioterapia neoadyuvante en cáncer de mama: correlación con el fenotipo molecular [Evaluation of pathological response to neoadjuvant chemotherapy in breast cancer: correlation with molecular phenotype]. Rev Esp Patol. 2021;54(1):8-16. doi:10.1016/j.patol.2020.07.003

8. Ogston KN, Miller ID, Payne S, et al. A new histological grading system to assess response of breast cancers to primary chemotherapy: prognostic significance and survival. Breast. 2003;12:320-327.

9. WHO. Rolling updates for breast cancer. https://www.who.int/news-room/fact-sheets/detail/breastcancer (accessed May 30, 2021)

10. Pathak M, Dwivedi SN, Deo SVS, Thakur B, Sreenivas V, Rath GK. Neoadjuvant chemotherapy regimens in treatment of breast cancer: a systematic review and network meta-analysis protocol. 
Syst Rev. 2018;7(1):89. Published 2018 Jun 26. doi:10.1186/s13643-018-0754-1

11. Weiss A, Bashour SI, Hess K, Thompson AM, Ibrahim NK. Effect of neoadjuvant chemotherapy regimen on relapse-free survival among patients with breast cancer achieving a pathologic complete response: an early step in the de-escalation of neoadjuvant chemotherapy. Breast Cancer Res. 2018;20(1):27. Published 2018 Apr 16. doi:10.1186/s13058-018-0945-7

12. Penault-Llorca F, Abrial C, Raoelfils I, et al. Comparison of the prognostic significance of Chevallier and Sataloff's pathologic classifications after neoadjuvant chemotherapy of operable breast cancer. Hum Pathol. 2008;39(8):1221-1228. doi:10.1016/j.humpath.2007.11.019

13. Bonnefoi H, Litière S, Piccart $M$, et al. Pathological complete response after neoadjuvant chemotherapy is an independent predictive factor irrespective of simplified breast cancer intrinsic subtypes: a landmark and two-step approach analyses from the EORTC 10994/BIG 1-00 phase III trial. Ann Oncol. 2014;25(6):1128-1136. doi:10.1093/annonc/mdu118

14. Fragomeni SM, Sciallis A, Jeruss JS. Molecular Subtypes and Local-Regional Control of Breast Cancer. Surg Oncol Clin N Am. 2018;27(1):95-120. doi:10.1016/j.soc.2017.08.005

15. Shintia C, Endang H, Diani K. Assessment of pathological response to neoadjuvant chemotherapy in locally advanced breast cancer using the Miller-Payne system and TUNEL. Malays J Pathol. 2016;38(1):25-32.

16. Untch M, Konecny GE, Paepke S, von Minckwitz G. Current and future role of neoadjuvant therapy for breast cancer. Breast. 2014;23(5):526-537. doi:10.1016/j.breast.2014.06.004

17. Omranipour R, Jalili R, Yazdankhahkenary A, Assarian A, Mirzania M, Eslami B. Evaluation of Pathologic Complete Response (pCR) to Neoadjuvant Chemotherapy in Iranian Breast Cancer Patients with Estrogen Receptor Positive and HER2 Negative and impact of predicting variables on pCR. Eur J Breast Health. 2020;16(3):213-218. Published 2020 Jul 1. doi:10.5152/ejbh.2020.5487

18. Bhargava R, Beriwal S, Dabbs DJ, et al. Immunohistochemical surrogate markers of breast cancer molecular classes predicts response to neoadjuvant chemotherapy: a single institutional experience with 359 cases [published correction appears in Cancer. 2011 May 15;117(10):2238]. Cancer. 2010;116(6):1431-1439. doi:10.1002/cncr.24876

19. Buzdar AU, Ibrahim NK, Francis D, et al. Significantly higher pathologic complete remission rate after neoadjuvant therapy with trastuzumab, paclitaxel, and epirubicin chemotherapy: results of a randomized trial in human epidermal growth factor receptor 2-positive operable breast cancer. J Clin Oncol. 2005;23(16):3676-3685. doi:10.1200/JC0.2005.07.032

20. Valachis A, Mauri D, Polyzos NP, Chlouverakis G, Mavroudis D, Georgoulias V. Trastuzumab combined to neoadjuvant chemotherapy in patients with HER2-positive breast cancer: a systematic review and meta-analysis. Breast. 2011;20(6):485-490. doi:10.1016/j.breast.2011.06.009

21. Wang C, Chen J, Xu X, et al. Dual HER2 Blockade in Neoadjuvant Treatment of HER2+ Breast Cancer: A Meta-Analysis and Review. Technol Cancer Res Treat. 2020;19:1533033820960721. doi:10.1177/1533033820960721 
22. Takada M, Toi M. Neoadjuvant treatment for HER2-positive breast cancer. Chin Clin Oncol. 2020;9(3):32. doi:10.21037/cco-20-123

23. Sharma P, Connolly RM, Roussos Torres ET, Thompson A. Best Foot Forward: Neoadjuvant Systemic Therapy as Standard of Care in Triple-Negative and HER2-Positive Breast Cancer. Am Soc Clin Oncol Educ Book. 2020;40:1-16. doi:10.1200/EDBK_281381

24. Sharma P, López-Tarruella S, García-Saenz JA, et al. Pathological Response and Survival in TripleNegative Breast Cancer Following Neoadjuvant Carboplatin plus Docetaxel. Clin Cancer Res. 2018;24(23):5820-5829. doi:10.1158/1078-0432.CCR-18-0585

25. Von Minckwitz G, Schneeweiss A, Loibl S, et al. Neoadjuvant carboplatin in patients with triplenegative and HER2-positive early breast cancer (GeparSixto; GBG 66): a randomised phase 2 trial. Lancet Oncol. 2014;15(7):747-756. doi:10.1016/S1470-2045(14)70160-3

26. Collins PM, Brennan MJ, Elliott JA, et al. Neoadjuvant chemotherapy for luminal a breast cancer: Factors predictive of histopathologic response and oncologic outcome [published online ahead of print, 2020 Dec 9]. Am J Surg. 2020;S0002-9610(20)30776-5. doi:10.1016/j.amjsurg.2020.11.053

27. Haque W, Verma V, Hatch S, Suzanne Klimberg V, Brian Butler E, Teh BS. Response rates and pathologic complete response by breast cancer molecular subtype following neoadjuvant chemotherapy. Breast Cancer Res Treat. 2018;170(3):559-567. doi:10.1007/s10549-018-4801-3

28. Wang J, Sang D, Xu B, et al. Value of Breast Cancer Molecular Subtypes and Ki67 Expression for the Prediction of Efficacy and Prognosis of Neoadjuvant Chemotherapy in a Chinese Population. Medicine (Baltimore). 2016;95(18):e3518. doi:10.1097/MD.0000000000003518

29. Tao M, Chen S, Zhang X, Zhou Q. Ki-67 labeling index is a predictive marker for a pathological complete response to neoadjuvant chemotherapy in breast cancer: A meta-analysis. Medicine (Baltimore). 2017;96(51):e9384. doi:10.1097/MD.0000000000009384

30. Samiei S, van Nijnatten TJA, de Munck L, et al. Correlation Between Pathologic Complete Response in the Breast and Absence of Axillary Lymph Node Metastases After Neoadjuvant Systemic Therapy. Ann Surg. 2020;271(3):574-580. doi:10.1097/SLA.0000000000003126

31. Díaz-Casas SE, Castilla-Tarra JA, Pena-Torres E, et al. Pathological Response to Neoadjuvant Chemotherapy and the Molecular Classification of Locally Advanced Breast Cancer in a Latin American Cohort. Oncologist. 2019;24(12):e1360-e1370. doi:10.1634/theoncologist.2019-0300

32. Jarząb M, Stobiecka E, Badora-Rybicka A, et al. Association of breast cancer grade with response to neoadjuvant chemotherapy assessed postoperatively. Pol J Pathol. 2019;70(2):91-99. doi:10.5114/pjp.2019.87101 